\title{
Ipl1/Aurora B kinase coordinates synaptonemal complex disassembly with cell cycle progression and crossover formation in budding yeast meiosis
}

\author{
Philip Jordan, ${ }^{1}$ Alice Copsey, ${ }^{1}$ Louise Newnham, ${ }^{1}$ Elizabeth Kolar, ${ }^{2,3}$ Michael Lichten, ${ }^{2}$ \\ and Eva Hoffmann ${ }^{1,4}$ \\ ${ }^{1}$ Medical Research Council Genome Damage and Stability Centre, University of Sussex, Falmer BN1 9RQ, United Kingdom; \\ ${ }^{2}$ Laboratory of Biochemistry and Molecular Biology, Center for Cancer Research, National Cancer Institute, Bethesda, Maryland \\ 20892, USA
}

\begin{abstract}
Several protein kinases collaborate to orchestrate and integrate cellular and chromosomal events at the G2/M transition in both mitotic and meiotic cells. During the G2/M transition in meiosis, this includes the completion of crossover recombination, spindle formation, and synaptonemal complex (SC) breakdown. We identified Ipl1/ Aurora B kinase as the main regulator of SC disassembly. Mutants lacking Ipl1 or its kinase activity assemble SCs with normal timing, but fail to dissociate the central element component Zip1, as well as its binding partner, Smt3/SUMO, from chromosomes in a timely fashion. Moreover, lack of Ipl1 activity causes delayed SC disassembly in a $c d c 5$ as well as a CDC5-inducible ndt80 mutant. Crossover levels in the ip11 mutant are similar to those observed in wild type, indicating that full SC disassembly is not a prerequisite for joint molecule resolution and subsequent crossover formation. Moreover, expression of meiosis I and meiosis II-specific B-type cyclins occur normally in ipl1 mutants, despite delayed formation of anaphase I spindles. These observations suggest that Ipl1 coordinates changes to meiotic chromosome structure with resolution of crossovers and cell cycle progression at the end of meiotic prophase.
\end{abstract}

[Keywords: Meiosis; synaptonemal complex; chromosome structure; Ipl1/Aurora B kinase; Cdc5/Polo-like kinase] Supplemental material is available at http://www.genesdev.org.

Received April 21, 2009; revised version accepted August 3, 2009.

The meiotic cell cycle consists of a single round of DNA replication followed by two consecutive nuclear divisions, named meiosis I and meiosis II. At the first meiotic division, homologous chromosomes (homologs) segregate to opposite spindle poles, mediated in part by chiasmata that provide a physical link between the homologs. At meiosis II, sister chromatids separate, analogous to a mitotic division, resulting in the formation of haploid gametes (Petronczki et al. 2003).

Several modifications to chromosome structure and behavior precede meiosis I. Sister chromatids shorten and form a "core" from which loops of chromatin emanate and on which axial element components assemble during leptotene. As the axial elements of the two homologs become stably juxtaposed ( $\sim 100 \mathrm{~nm})$ by the deposition of

\footnotetext{
${ }^{3}$ Present address: Biochemistry, Cellular, and Molecular Biology Graduate Program, Johns Hopkins University, 1830 E. Monument St., Suite 2-102, Baltimore, MD 21205, USA.

${ }^{4}$ Corresponding author.

E-MAIL eh58@sussex.ac.uk; FAX 44-01273-877121.

Article is online at http://www.genesdev.org/cgi/doi/10.1101/gad.536109.
}

transverse filament(s) at zygotene, they form the lateral elements of the morphologically conserved synaptonemal complex (SC) (Roeder 1997; Zickler and Kleckner 1999). By pachytene, homologous chromosomes are synapsed along their entire length and the chromosomes are highly compacted. Upon pachytene exit, the chromatin decondenses and the SC is disassembled as cells enter the diffuse stage. At diplotene, most of the SC has been removed, centrosomes are separated, and chiasmata become cytologically visible. Eventually, metaphase I spindles are formed as cells prepare for the first nuclear division.

Budding yeast has a single transverse filament protein, termed Zip1 (Sym et al. 1993; Sym and Roeder 1995) - the structural equivalent of SYCP1/SYP-1 in mammals and nematodes, c(3)g in Drosophila, and ZYP-1 in Arabidopsis (Colaiacovo 2006; Bhalla and Dernburg 2008). Initiation of Zip1 deposition along chromosome cores occurs from centromeres (Tsubouchi and Roeder 2005; Tsubouchi et al. 2008) as well as from future sites of crossover formation (the precursors to chiasmata) along chromosome arms (Roeder 1997; Henderson and Keeney 2005). 
At centromeres, Zip1 protects meiotic cells from the formation of aneuploid spores, since zip1 mutants show increased crossover formation near centromeres (Chen et al. 2008) and such crossovers are associated with the precocious separation of sister chromatids at meiosis I (Rockmill et al. 2006). Furthermore, Zip1 also promotes centromere tethering, and thereby accurate segregation of chromosome pairs that fail to cross over (L Newnham, P Jordan, B Rockmill, S Roeder, and E Hoffmann, in prep.).

Along chromosome arms, crossover recombination is initiated from a subset of meiotic recombination events induced by the introduction of double-strand breaks (DSBs). In budding yeast, the differentiation of DSBs to yield crossovers or noncrossovers is imposed prior to or concomitant with strand exchange (Allers and Lichten 2001; Hunter and Kleckner 2001). Moreover, crossovers are preceded by specific DSB repair intermediates, including single-end invasions (SEIs) and joint molecules (Schwacha and Kleckner 1995; Allers and Lichten 2001; Hunter and Kleckner 2001). Consistent with SC components being important for the initiation of crossover recombination, SC mutants show a decrease in the levels of SEIs (Borner et al. 2004) as well as subsequent intermediates and crossover products (Sym et al. 1993; Storlazzi et al. 1996; Borner et al. 2004). However, the role of Zipl at sites of crossovers is unclear. End-to-end polymerization of Zip1 along chromosomes is not required to promote crossovers in certain mutant backgrounds (Storlazzi et al. 1996; Hooker and Roeder 2006), suggesting that SC nucleation may be sufficient to facilitate the molecular transitions during crossover initiation (Storlazzi et al. 1996).

Zip1 has been proposed to bind sumoylated proteins along the lateral elements (Cheng et al. 2006; Hooker and Roeder 2006). As recombination proceeds and linear Zip1-SUMO stretches are formed, chromosomes progressively condense, culminating in highly compacted "worm-like" structures by pachytene. Both DSB repair and SC formation are monitored by checkpoints that prevent pachytene exit, the first step in the G2/M progression, until these processes are complete (Roeder and Bailis 2000; Wu and Burgess 2006). Pachytene exit is regulated by the $\mathrm{Ndt} 80$ transcription factor that controls the expression of $\sim 200$ genes leading to cell cycle progression (Xu et al. 1995; Chu and Herskowitz 1998; Chu et al. 1998).

The first cytological hallmark of pachytene exit is the disassembly of the SC. We showed recently that Zip1 dissociates from chromosome arms, but remains at centromeres, after SC disassembly. By diplotene, Zip1 foci colocalize with Ctf19-13Myc, a centromere marker, suggesting that Zip1 is retained specifically at centromeres. Zip1 is also visible on metaphase I spindles, then eventually localizes to the poles of anaphase I spindles together with Ctf19-13Myc (L Newnham, P Jordan, B Rockmill, S Roeder, and E Hoffmann, in prep.). Thus, Zip1 likely remains associated with centromeric regions after SC disassembly. It is unknown what facilitates the removal of Zip1 from arm regions and how Zip1 is protected (or reloaded) at centromeres at the G2/M transition.
SC disassembly is coordinated with other chromosomal and cellular events, including the appearance of crossover products; the separation of the microtubule organizing centers, known as spindle pole bodies (SPBs) in budding yeast; and modifications to kinetochores that ensure that sister kinetochores orient toward the same spindle pole at meiosis I (Toth et al. 2000). In budding yeast, ndt80 mutants arrest with unseparated SPBs, unresolved joint molecules, and full SCs (Xu et al. 1995; Allers and Lichten 2001). Timely SC disassembly as well as joint molecule resolution can be rescued by the expression of a single gene: Cdc5/Polo-like kinase (Sourirajan and Lichten 2008). Clb1, Clb3, Clb4, and Clb6, B-type cyclin components of mitotic cyclin-dependent kinase (CDK$\mathrm{M})$, which are also regulated by Ndt80, are presumably still required for SPB separation and entry to $M$ phase, because these events are still inhibited (Sourirajan and Lichten 2008).

The mechanism of coordination between joint molecule resolution into crossovers and SC disassembly remains unclear. $c d c 5$ mutants fail to resolve the majority of joint molecules but show a less pronounced SC disassembly defect (Clyne et al. 2003). Thus, although Cdc5 is sufficient to mediate SC disassembly in ndt80s mutants (Sourirajan and Lichten 2008), it is unclear whether it is required to do so in normal meiosis.

Unlike Cdc5 and CDK1-M, Ipl1/Aurora B kinase, a chromosomal passenger protein, is not regulated by $\mathrm{Ndt} 80$ and is expressed maximally between 3 and $6 \mathrm{~h}$ in meiosis, at the G2/M transition (Chu 1998; Monje-Casas et al. 2007). These proteins regulate kinetochoremicrotubule attachments, spindle formation, and cytokinesis (Ruchaud et al. 2007; Kelly and Funabiki 2009), as well as chromosome dynamics at the G2/M transition (Hsu et al. 2000). Characterization of Ipll activity during meiosis has focused on its role in chromosome segregation. Together with the monopolin complex that ensures the cosegregation of sister kinetochores to the same spindle pole at meiosis I (Toth et al. 2000), Ipll promotes accurate homolog segregation at the first meiotic division (Monje-Casas et al. 2007). In addition, Ipl1 also ensures the retention of protein phosphatase $2 \mathrm{~A}$ (PP2A) at kinetochores. PP2A protects Sgol and thereby centromeric cohesin, which must be retained until meiosis II to prevent precocious sister chromatid separation at meiosis I (Riedel et al. 2006; Tang et al. 2006; Yu and Koshland 2007).

Ipl1 localizes as distinct foci on meiotic chromosomes throughout meiotic prophase I in budding yeast and localizes to kinetochores by metaphase I (Monje-Casas et al. 2007; Yu and Koshland 2007). In mouse spermatocytes, another chromosomal passenger complex protein, INCENP, shows confluent colocalization with the central element component SYCP1 from zygotene to pachytene, before relocating to the centromeres at late pachytene (Parra et al. 2003, 2009). Although Aurora B kinase staining is detected only from diplotene onward (Parra et al. 2003), inhibition of Aurora B kinase with ZM447439 blocks dissociation of the lateral element component SYCP3. However, the dissociation of SYCP1, the central element, is not affected (Sun and Handel 2008). 
Here, we investigate Ipll's role in SC assembly and disassembly in budding yeast. Cytological and biochemical observations show that Ipl1 promotes the timely disassembly of the SC prior to diplotene and the G2/M transition. Crossover levels are not decreased in the ipl1 mutant and cell cycle progression occurs with similar kinetics to the wild-type strain. These observations support the conclusion that SC disassembly, promoted by Ipl1, occurs in parallel with cell cycle progression and maturation of crossover products.

\section{Results}

SC disassembly and Zip1 degradation are delayed in ipl1-mn mutants.

Ipl1 is expressed throughout meiosis, although at a lower level compared with mitotic cells (Monje-Casas et al. 2007). To investigate whether Ipl1 affects SC disassembly, we used a strain where IPL1 expression is regulated by the $C L B 2$ promoter, which is down-regulated during meiosis

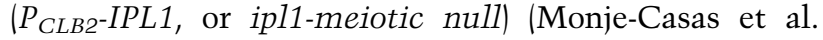
2007). 3HA-Ipl1 levels decrease in the ipl1-mn strain upon entry into meiosis and Ipll kinase activity becomes undetectable compared with the wild-type strain, as assessed by phosphorylation of H3 Ser10 during meiotic prophase I (Supplemental Fig. S1). Using the ipl1-mn and an isogenic wild-type strain, we assessed surface spread nuclei for Zip1 staining (Fig. 1A). In the wild type, foci of Zip1 (leptotene) were observed in one-third of the nuclei by $2 \mathrm{~h}$, short linear structures of Zip1 interspersed with Zip1 foci ("dot-linear," zygotene) appeared around $4 \mathrm{~h}$, and nuclei consisting entirely of linear Zipl staining (pachytene) were observed from $5 \mathrm{~h}$. In the ipl1-mn mutant, SC assembly occurred similarly (Fig. 1B,C), although we observed a delay in the appearance of nuclei with punctate Zip1 from the 2- to 3-h time point in the ipl1-mn mutant. Subsequent analyses showed that other meiotic events, such as DNA replication, DSB formation, and the nuclear divisions, were also delayed (see below), suggesting a modest defect in the initiation of meiosis, as reported previously (Monje-Casas et al. 2007). Thus, the slight delay in SC assembly is most likely due to a general delay in meiotic progression.

Accumulation of Zip1 aggregates (polycomplexes, PCs), are observed in a large proportion of nuclei from mutants with defective SC assembly (Roeder 1997; Zickler and Kleckner 1999). However, in both the wild-type and

A
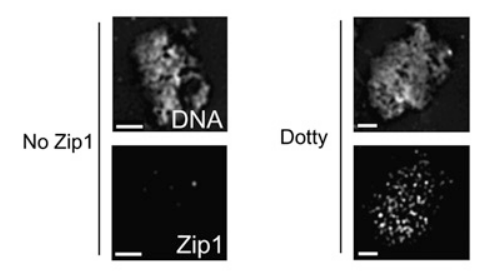
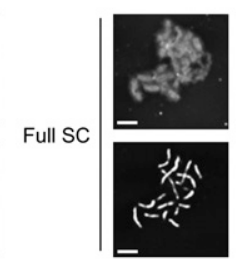

B

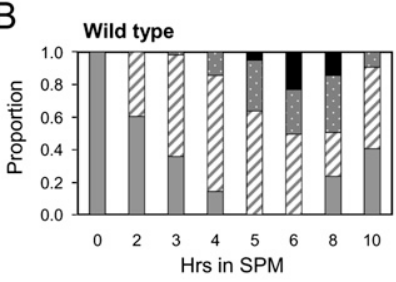

C

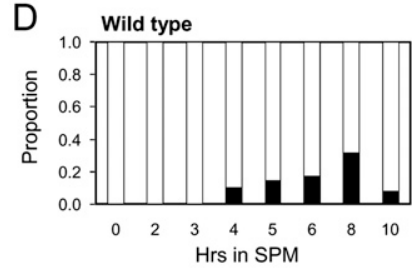

E
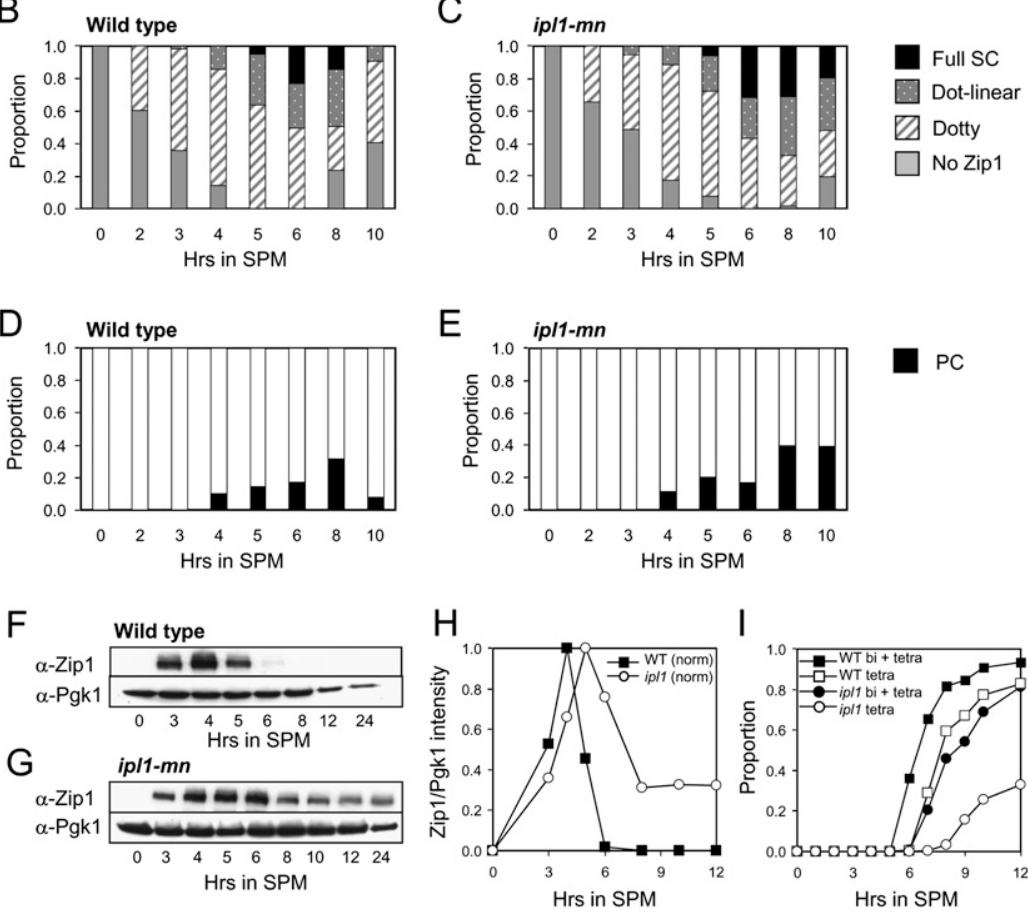

I

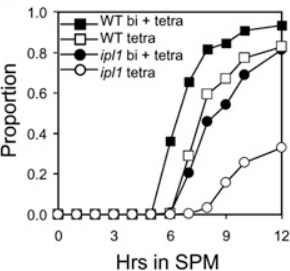

Figure 1. SC disassembly and Zip1 degradation are delayed in ipl1-mn mutants. (A) Examples of Zip1 staining. $(B, C)$ Proportion of nuclei with dotty or punctate, dot-linear, linear Zip1, and no staining in wild type $(\mathrm{Y} 940, B)$ and ipl1-mn (Y1206, C). More than 50 nuclei were scored for each time point. $(D, E)$ Proportion of nuclei with Zip1 aggregates $(\mathrm{PC}) .(F, G)$ Western blot analysis of Zip1 appearance and disappearance in wildtype $(F)$ and ip11-mn $(G)$ cells in a separate experiment. $(H)$ Ratios of Zip1/Pgk1 levels normalized to the maximum Zip1/Pgk1 ratio (Materials and Methods). (I) Completion of the two meiotic nuclear divisions. Cells were assessed whether they were binucleate (meiosis I) or tetranucleate (meiosis II). In a proportion of the ipl1-mn nuclei, the DNA looked "stretched," suggesting that metaphase I-anaphase I transition was delayed (Monje-Casas et al. 2007). Subsequent analysis demonstrated that these "stretched" nuclei contained long metaphase I/early anaphase I spindles (Supplemental Fig. S2). Nevertheless, by $24 \mathrm{~h}$, $99 \%$ of wild-type and $88 \%$ of ipl1-mn cells had completed at least one meiotic nuclear division. 
Jordan et al.

ipl1-mn mutant, PCs were observed in only $\sim 10 \%-20 \%$ of nuclei during early time points $(2-5 \mathrm{~h}$ in sporulation medium, SPM) (Fig. 1D,E), indicating that SC assembly occurred normally.

In the wild type, the proportion of nuclei with entirely linear Zip1 increased to $\sim 25 \%$ by $6 \mathrm{~h}$ before decreasing in frequency at $8 \mathrm{~h}$. By $10 \mathrm{~h}$, no such nuclei were detected (Fig. 1B). This loss is interpreted as exit from the pachytene stages and coincides with an increase in surface spread nuclei containing PCs of Zip1 ( 35\%). These observations are consistent with electron micrograph studies that show a single dense body stained in nuclei with separated SPBs, indicative of diplotene (Zickler and Olson 1975). We find that the Zipl PCs are often juxtaposed to duplicated SPBs lying side-by-side (Fig. 2A, "diffuse stage").

The decrease in the frequency of nuclei with full SCs by $8 \mathrm{~h}$ was not observed in the ipl1-mn mutant. Even at $10 \mathrm{~h}, \sim 20 \%$ of nuclei displayed linear Zipl staining. Thus, Zip1 is observed as linear stretches for an extended period in the absence of Ipll. One interpretation of this observation is that the pachytene period is extended.
An extended persistence of nuclei with mature SC could be indicative of pachytene arrest or a delay in SC disassembly. To distinguish between the two possibilities, we followed the degradation of Zip1 and the nuclear divisions. Upon pachytene exit, Zip1 degradation occurred rapidly in the wild-type strain as cells entered the two nuclear divisions (Fig. 1F-I). In the ipl1-mn mutant, some Zip1 protein was degraded; however, a substantial fraction $(\sim 30 \%-40 \%)$ was retained (from 8 to 24 h), even as the number of cells that had completed at least one meiotic nuclear division doubled in the same time period (Fig. 1I). These observations are consistent with the hypothesis that SC disassembly is delayed.

Finally, in the ipl1-mn mutant, we noticed that a significant proportion of binucleate cells ( 20\%-30\%) appeared "stretched," rather than having properly divided their DNA mass. We examined this further and observed that these cells contained long metaphase I/early anaphase I spindles (Supplemental Fig. S2). This is consistent with previous observations that the transition from short metaphase I to anaphase I spindles is delayed in the ipl1mn mutant (Monje-Casas et al. 2007). A similar phenomenon was also observed at metaphase II/anaphase II
A

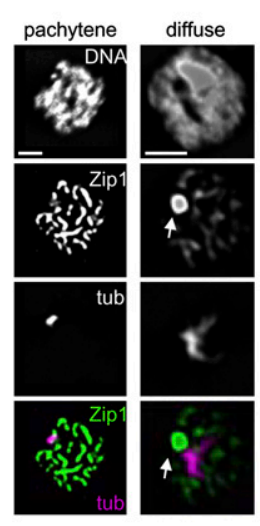

Wild type

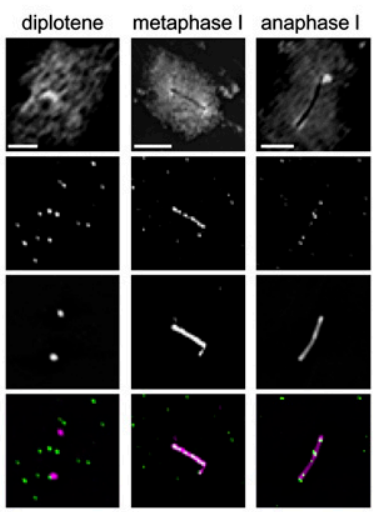

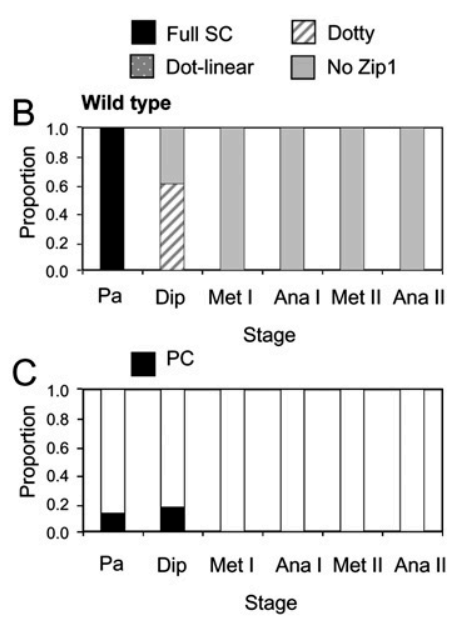

Figure 2. SC disassembly and cell cycle progression are decoupled in ipl1-mn cells. Surface spread nuclei were stained for Zip1 (green), tubulin (magenta), and DNA. $(A, D)$ Examples of wild-type (Y1381) and ipl1-mn (Y1669) nuclei at pachytene, diffuse, diplotene, metaphase I, and anaphase I stages. The arrows indicate PCs of Zip1. (tub) Tubulin. Bars, $2 \mu \mathrm{m}$. $(B, E)$ Quantification of Zipl staining based on cell cycle stage (as determined by tubulin staining). (Pa) Pachytene (single tubulin focus); (Dip) diplotene (two tubulin foci); (Met I) metaphase I (single short thick spindle); (Ana I) anaphase I (single elongated spindle); (Met II) metaphase II (two short thick spindles); (Ana II) anaphase II (two elongated spindles). $(C, F)$ Proportion of nuclei with PC. For each stage, 30-50 nuclei were scored.

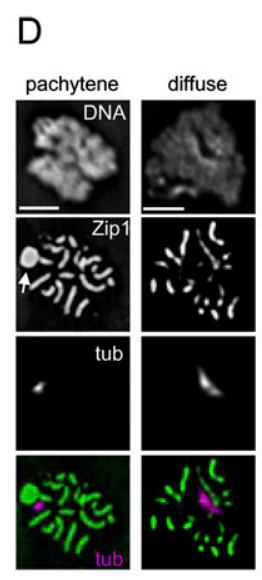

ipl1-mn
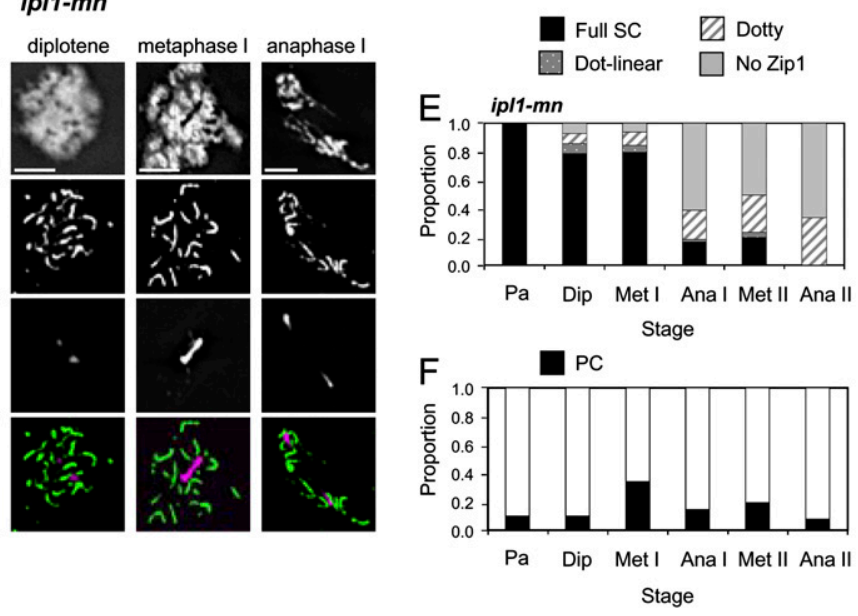
(Supplemental Fig. S2). The stretched nuclear phenotype is not alleviated by deletion of ZIP1, since an ipl1-mn zip1 double mutant also displayed a stretched nuclear phenotype. If anything, both the double and the zip1 single mutant displayed a more pronounced phenotype (Supplemental Fig. S3).

\section{SC disassembly and spindle formation are decoupled in ipl1-mn}

If SC disassembly is incomplete or delayed, but the meiotic nuclear divisions occur with only a short delay relative to wild type (Fig. 1I; Supplemental Fig. S2), then SC disassembly and the cell cycle must be decoupled. This hypothesis predicts that cells should be observed in which the SCs are still assembled despite progression of spindle behavior to diplotene or beyond. To test whether this was the case, we stained surface spread nuclei with antibodies against both tubulin and Zip1 (Fig. 2). In wild type, the SCs had disassembled by diplotene and only foci of Zip1 were present (Fig. 2A,B). We showed recently that these foci localize to centromeres (L Newnham, P Jordan, B Rockmill, S Roeder, and E Hoffmann, in prep.). By metaphase I, Zip1 was observed predominantly on the metaphase I spindle (Fig. 2A,B).

In contrast to the wild-type cells, $\sim 80 \%$ of ipl1-mn mutant nuclei with separated SPBs or metaphase I spindles displayed linear Zip1 staining (Fig. 2D,E). Since SPB separation and metaphase I spindle formation depend on CDK1-M activity (Xu et al. 1995; Sourirajan and Lichten 2008), these results support the conclusion that SC disassembly and cell cycle progression have become decoupled in the absence of Ipl1.

\section{Meiotic prophase I is not contracted in ipl1-mn}

The decoupling of SC disassembly and spindle behavior shows that SC disassembly occurs in parallel with cell cycle control. The delayed SC disassembly could be due to Ipll regulating the timing of meiotic prophase I or SC disassembly per se. To distinguish between these possibilities, we assessed the progression of the meiotic cell cycle in the ipl1-mn mutant by analyzing the kinetics of premeiotic S-phase completion and SPB separation, the last stage of meiotic prophase, as well as the expression of two B-type cyclins-the meiosis I-specific Clb1 and meiosis II-specific Clb3 (Carlile and Amon 2008). Although premeiotic $S$ phase was initiated at time points similar to the wild-type strain (2-3 h), completion was delayed by $\sim 30-60 \mathrm{~min}$ (Fig. 3A; data not shown). SPB separation was similarly delayed by $\sim 30-60 \mathrm{~min}$ compared with the wild type (Fig. 3B), indicating that meiotic prophase I is not contracted in the ipl1-mn mutant, consistent with previous observations (Monje-Casas et al. 2007).

To determine whether a contraction in meiotic prophase I is sufficient to cause a SC disassembly phenotype, we assessed SC disassembly in a mad3D mutant, which halves the duration of meiotic prophase I (Cheslock et al. 2005). Nevertheless, SC disassembly occurs prior to metaphase I (zero of 30 metaphase I nuclei contained linear SCs) (data not shown), consistent with the interpretation that contraction of prophase I is insufficient to decouple SC disassembly from cell cycle progression.

Finally, we assessed whether the expression of B-type cyclins was affected in the ipl1-mn mutant. To this end, we used a strain where Ndt80 expression was shut off, but was inducible by addition of $\beta$-estradiol (NDT80-IN) (Benjamin et al. 2003; Carlile and Amon 2008). Using this system, $>80 \%$ of nuclei from both the wild-type and the ip11-mn strains showed full SCs prior to release (data not shown). Nevertheless, Zip1 disappearance and SC breakdown were consistently delayed in ipl1-mn relative to wild type upon Ndt80 expression. Expression of the meiosis I-specific B-type cyclin Clb1-13Myc (Carlile and Amon 2008) was similar in the ipl1-mn and wild-type strains (Fig. 3C). Thus, the transition from meiotic prophase to the first meiotic division appeared to occur normally in the ipl1-mn mutant.

Clb3-13Myc expression is confined to meiosis II (Carlile and Amon 2008) and occurred with similar timing in the ipl1-mn and the wild-type strains (Fig. 3D), despite the finding that anaphase I spindles appear to persist (Monje-Casas et al. 2007) and that a significant proportion of ipl1-mn nuclei showed progression defects from metaphase I-anaphase I "stretched" DNA phenotype) (Supplemental Fig. S4). However, the nuclear division profile in the Clb3-13Myc time course shows that the initiation of the second nuclear division, which gives rise to a high proportion of stretched nuclei in the ipl1$m n$, nevertheless occurred with similar timing as in the wild-type strain (Supplemental Fig. S4). Thus, although the completion of both meiotic nuclear divisions was delayed in the ipl1-mn strain, initiation thereof and Clb3$13 \mathrm{Myc}$ expression occurred with timing similar to the wild-type strain.

\section{Ipl1 kinase activity is important for SC disassembly}

Ipl1 is an ATP-dependent kinase whose ATP-binding domain can be mutated to accommodate a larger nucleoside analog that inhibits its activity (ip11-M181G,T244A, aka ipl1-as5) (Pinsky et al. 2006). Inhibitors include 1-NA-PP1 (1-[1,1-dimethylethyl]-3-[1-naphthalenyl]-1Hpyrazolo[3, 4-d]pyrimidin-4-amine) and generally do not affect other, unmodified ATP-dependent kinases (Bishop et al. 2000). We inhibited ip11-as5 cells with 1-NA-PP1 after SC assembly, but prior to the onset of SC disassembly, by arresting cells in pachytene (at $5.5 \mathrm{~h}$ ) and then releasing cells using the NDT80-IN $(6 \mathrm{~h})$. The 1-NA-PP1treated ipl1-as5 cells recapitulated the ipl1-mn phenotype (Supplemental Fig. S5), whereas mock treatment (DMSO alone) had no effect (data not shown). A slightly milder effect of the ip11-as5 allele compared with ipl1-mn was observed; this is likely due to a subset of cells not being exposed to sufficient levels of inhibitor. In diplotene and metaphase I nuclei, $32 \%$ and $30 \%$ contained full SCs compared with $40 \%$ in ipl1-mn (data not shown). These observations support the conclusion that Ipll promotes de facto SC disassembly and that it requires its kinase activity to do so. Whether or not Ipll phosphorylates 
Jordan et al.

Figure 3. Cell cycle progression and crossover formation are relatively normal in the ipl1-mn mutant. (A) FACS analysis of premeiotic S-phase completion in wild type (Y940, left panel) and ipl1-mn (Y1206, right panel). (B) Proportion of cells with separated SPBs. More than 200 cells were scored per time point. $(C, D)$ Expression of B-type cyclins and nuclear divisions in NDT80-IN wild-type and ip11-mn strains. $(C)$ Expression levels of Clb1-13Myc, a meiosis I-specific B-type cyclin in wild type (Y1661, top panels) and ipl1-mn (Y1663, bottom panels). (D) Expression levels of Clb3-13Myc, a meiosis II-specific B-type cyclin in wild type (Y1581, top panels) and ipl1-mn (Y1582, bottom panels). Pgk1 was used as loading control. The arrow indicates release from pachytene arrest by induction of Ndt80. $(E, F)$ Southern blots of crossover formation at the URA3-ARG4 hot spot (Supplemental Fig. S7) in wild type (Y940, E) and ipl1-mn (Y1206, F). (P1, P2) Parental DNA molecules; (CO1, CO2) crossover products; (DSB1, DSB2) DSB products. Molecular weight markers (M) are a HinDIII digest of bacteriophage $\lambda$ DNA $(23.1,9.4,6.6,4.4,2.3$, and $2.2 \mathrm{~kb}$ from top to bottom). $(G, H)$ Quantification of DSBs and crossovers.
A

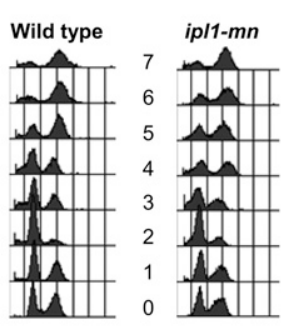

Hrs in SPM

C

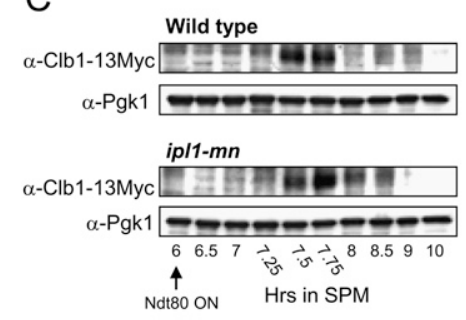

E

Wild type
Hrs in SPM

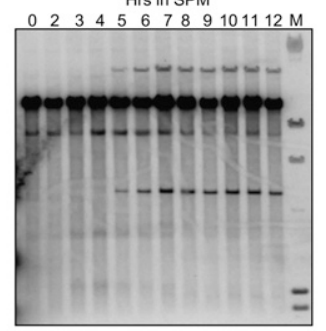

G

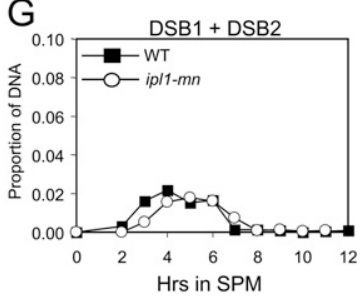

B

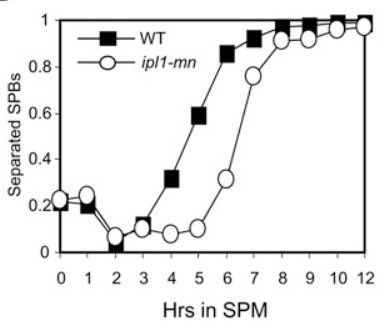

$\mathrm{D}$
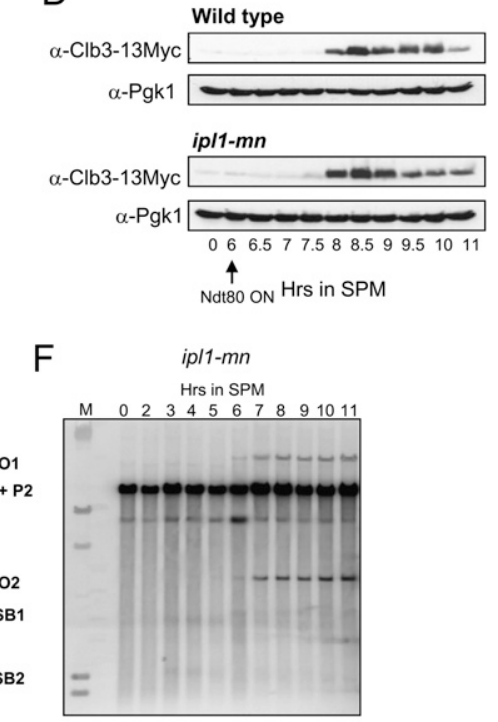

$\mathrm{H}$

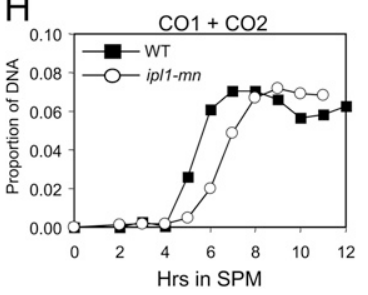

Zip1 remains to be determined. Although Zip1 is a phosphoprotein, its migration on one-dimensional PAGE remains unaffected in ipl1-mn mutants (Supplemental Fig. S6). We do note that simultaneous mutation of three potential Ipl1 phosphorylation consensus sites in Zip1 (ZIP1T273A, S274A, D381A, T382A, S511A, and T512A) affects neither SC assembly nor disassembly (data not shown).

\section{The DSB-crossover transition occurs normally} in the ipl1-mn mutant

The SC disassembly defect observed in the ipl1-mn mutant allowed us to address whether SC disassembly is a prerequisite for crossover formation. To this end, we analyzed meiotic crossing over at the URA3-ARG4 recombination hot spot inserted at the LEU2 (parent 1, P1) and HIS4 (parent 2, P2) loci, respectively, on the two parental Chromosomes III (Allers and Lichten 2001)
(Supplemental Fig. S7). DSBs were formed between 2 and $3 \mathrm{~h}$ in the wild-type strain (Fig. 3E,G) and were delayed $\sim 60 \mathrm{~min}$ in the ipl1-mn mutant (Fig. 3F,G). This is consistent with the similar delay in the completion of premeiotic $S$ phase, since replication timing determines the onset of DSB formation in budding yeast (Borde et al. 2000). Crossovers were formed between 4 and $5 \mathrm{~h}$ in the wild-type and 5 and $6 \mathrm{~h}$ in ipl1-mn strain (Fig. 3H). Thus, the time between DSB and crossover formation $(\sim 2 \mathrm{~h})$ was similar in both strains. Furthermore, final crossover levels were also similar in the ipl1-mn mutant compared with the wild-type strain $(\sim 7 \%)$ (Fig. $3 \mathrm{H})$. Finally, analysis of the levels of crossover and noncrossover products also failed to show any differences between the wild-type and the ipl1-mn mutant, except for the $\sim 60$-min delay (Supplemental Fig. S8). These observations support the conclusion that crossover formation and DSB repair, more generally, are not affected in the ipl1-mn mutant. Thus, 
the delayed and incomplete SC disassembly phenotype does not cause adverse effects on crossover formation.

\section{IPL1 promotes SC disassembly in cdc5-mn and CDC5-IN cells}

Recently, Cdc5 Polo-like kinase has been implicated in pachytene exit, promoting Holliday junction resolution and SC disassembly. The period in which pachytene nuclei are observed is increased in cdc5-mn mutants, similar to ipl1-mn cells (Brar et al. 2009). When analyzed by tubulin staining, SC disassembly is less affected in a cdc5-mn mutant compared with ipl1-mn. Fewer than $20 \%$ of diplotene nuclei contained full SCs, and by metaphase I, this was further decreased (4\%) (Fig. 4A,C). In the majority of nuclei at diplotene or beyond, Zip1 was often present as foci in the cdc5-mn mutant (Fig. 4A). These foci were more numerous than in wild type, where
Zip1 was retained at centromeres after pachytene exit (data not shown).

SC disassembly was similarly delayed in the ipl1-mn cdc5-mn double mutant compared with the ipl1-mn single mutant (Fig. 4B,D). Thirty-two percent of metaphase I nuclei contained full SCs and another $63 \%$ contained dot-linear Zip1 staining compared with $49 \%$ full SCs and 48\% dot-linear in ipl1-mn (data not shown), and with $4 \%$ full SCs and 36\% dot-linear in the cdc5-mn mutant (Fig. 4C). Furthermore, the dot-linear staining pattern of ipl1-mn and the cdc5-mn ipl1-mn double mutant contained more lines of Zip1 compared with cdc5-mn. Thus, $97 \%$ and $95 \%$ of metaphase I nuclei contained significant stretches of Zip1 in the ipl1-mn and cdc5-mn ipl1-mn mutants. These observations indicate that Ipl1 promotes SC disassembly in cdc5-mn mutants. Importantly, these experiments were carried out in the NDT80-IN background. We did not observe any progression
A
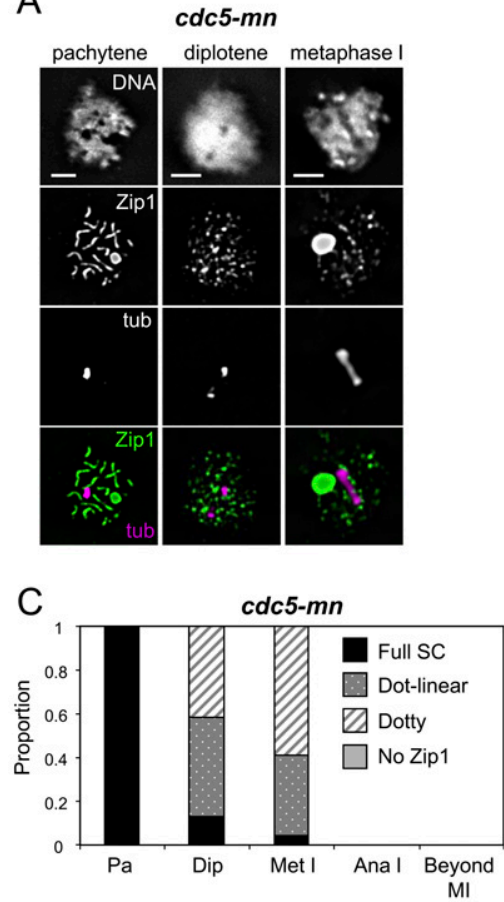

B

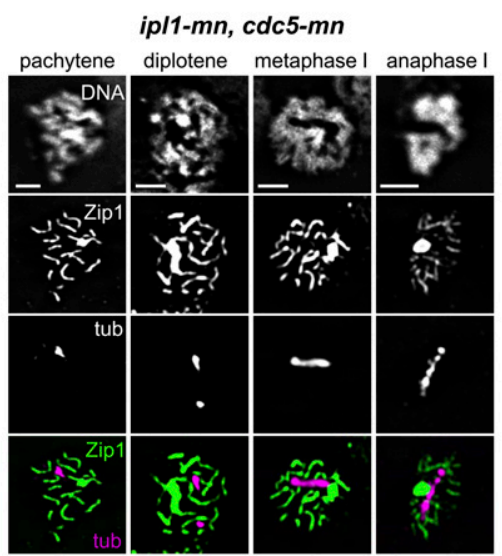

D

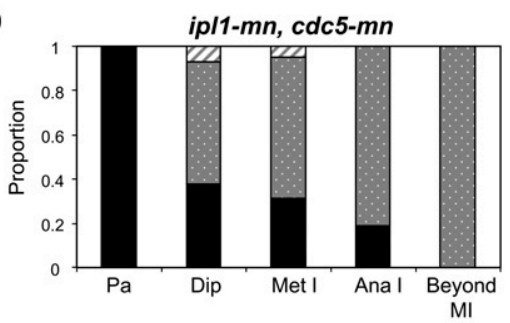

$\mathrm{F}$

-ED $i p / 1-m n, C D C 5-I N+E D$

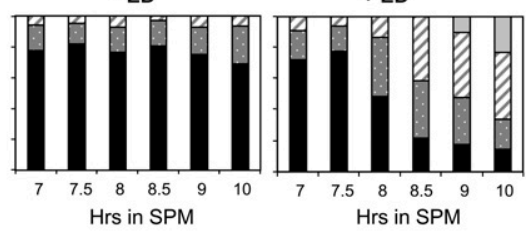

$\mathrm{Hrs}$ in SPM

$\mathrm{Hrs}$ in SPM

$\mathrm{Hrs}$ in SPM

Full SC $\square$ Dot-linear $\square$ Dotty $\square$ No Zip1
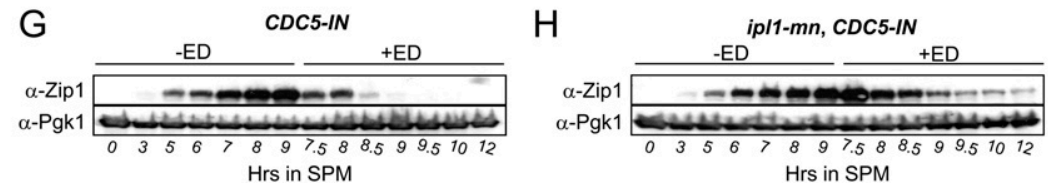

Figure 4. Ipll promotes SC disassembly in cdc5-mn and CDC5-IN cells. Examples of nuclei and quantification of Zip1 staining from cdc5-mn (Y1657, A,C) and cdc5-mn ipl1-mn (Y2030, $B, D)$ mutants. $(E, F)$ Analysis of Zip1 SC disassembly in a time course using a ndt80 $\Delta$ strain with inducible CDC5 (CDC5-IN, Y2262, E) expression by addition to $\beta$-estradiol $(+\mathrm{ED}$, at $7 \mathrm{~h})$ and in an $n d t 80 \Delta$ CDC5-IN ip1-mn strain (Y2263, F). At least 100 nuclei were scored for each stage. Zip1 protein levels are shown for ndt80A CDC5IN $(G)$ and ndt80D CDC5-IN ip1-mn $(H)$ strains. 
Jordan et al.

beyond pachytene in cdc5-mn NDT80-IN cells, where NDT80 expression was not induced by addition of $\beta$-estradiol (-ED) (Fig. 4E; data not shown). This suggests that CDC5 activity is extremely low, if present at all, in these cells.

Finally, expression of CDC5 in an ndt80 mutant (CDC5-IN) is sufficient to trigger SC disassembly (Fig. 4E,G; Sourirajan and Lichten 2008). We find that the ipl1$\mathrm{mn}$ allele delays SC disassembly in the CDC5-IN strain, suggesting that Ipl1 is important for SC disassembly regulated by $\mathrm{Cdc} 5$.

\section{Zip1 degradation is inhibited in MG-132-treated cells}

In wild type, Zip1 dissociation from meiotic chromosomes and its degradation occurred with similar timing after pachytene release (Fig. 5A,B). To address whether the two can be decoupled and whether the degradation of Zip1 depends on the 26S proteasome, we treated NDT80IN pdr5s cells with the $26 \mathrm{~S}$ proteasome inhibitor MG132 (Pdr5 is a multidrug transporter that pumps out MG132 and is dispensible for meiosis) (Carlile and Amon 2008; data not shown). Cells were arrested at pachytene, and treated with $20 \mu \mathrm{M}$ MG-132 or DMSO (5.5 h) before being released from arrest by induction of Ntd80 expression via addition of $\beta$-estradiol (6 h, "Ndt80 ON"). Additional MG-132 or DMSO was added at 1-h intervals ( 6.5 and $7.5 \mathrm{~h}$ ) before being washed out ( $8.5 \mathrm{~h}$ ) (Fig. 5E,G). In both mock-treated and MG-132-treated $p d r 5 \Delta$ cells, SC disassembly occurred with similar kinetics (Fig. 5F,H). Sixty minutes after Ndt80 induction, only $\sim 30 \%$ of nuclei showed full SCs, and this proportion decreased
Figure 5. Zip1 degradation and removal from meiotic chromosomes. Pachytene-arrested NDT80-IN cells were released by the addition of $\beta$-estradiol to induce Ndt80 expression after $6 \mathrm{~h}$ in SPM ("Ndt80 $\mathrm{ON}, "$ indicated by the arrow), and Zipl and Pgk1 protein levels as well as Zip1 dissociation from meiotic chromosomes were determined. Arrest and release in wild type (Y1602, $A, B)$ and ip11-mn $(Y 1538, C, D) .(E-H)$ Arrest-and-release experiment with $p d r 5 \Delta$ cells (Y1553) treated with DMSO only $(E, F)$ or $20 \mu \mathrm{M}$ MG-132 $(G, H)$ at the indicated time points $(+)$. $(I-L)$ SC disassembly and Zip1 degradation after release from pachytene arrest in $c d c 20$ $m n(Y 1656, I, J)$ and cdc20-mn ipl1-mn (Y1627, $K, L)$. More than 50 nuclei were scored for each time point.
A

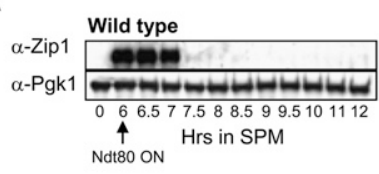

C

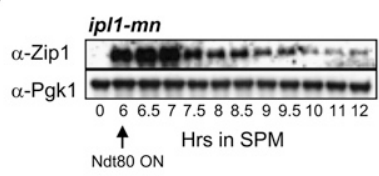

E

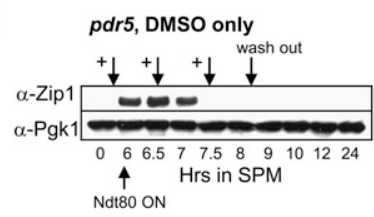

G

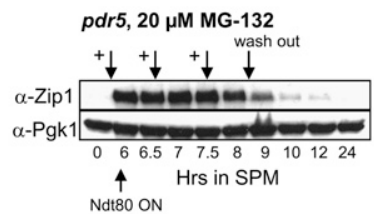

I

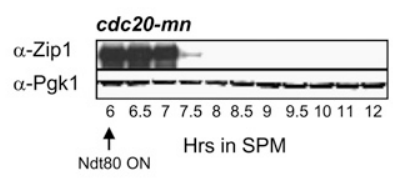

K

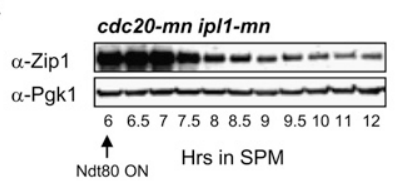

B
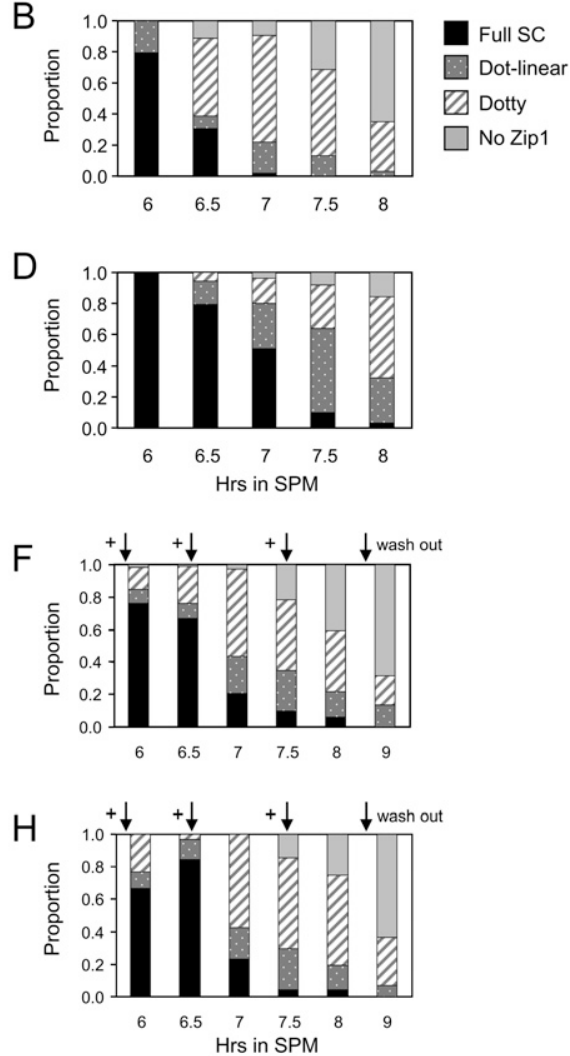

J

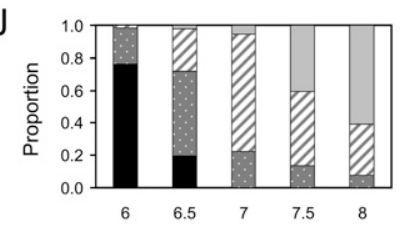

L

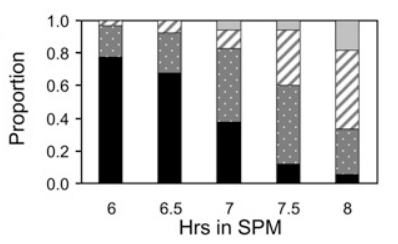


further with time, such that by $8 \mathrm{~h}$, Zip1 had dissociated from the majority of meiotic chromosomes and no PCs were observed (data not shown). While Zip1 protein disappeared from mock-treated cells within $1.5 \mathrm{~h}$ of $\mathrm{Ndt} 80$ induction, Zip1 protein levels in MG-132-treated cells remained high until removal of MG-132 at $8.5 \mathrm{~h}$. Zip1 protein levels then decreased $(9 \mathrm{~h})$ and eventually became undetectable $(24 \mathrm{~h})$ (Fig. 5E,G). Thus, in the presence of MG-132, Zip1 dissociated from meiotic chromosomes with normal timing but failed to be degraded. These observations show that Zip1 dissociation from chromosomes can occur independently of its degradation, as had been shown previously using ndt80 $\Delta$ cells expressing the Cdc5 kinase (Fig. 4E,G; Sourirajan and Lichten 2008). Our data further indicate that $\mathrm{Zip} 1$ degradation is dependent on the $26 \mathrm{~S}$ proteasome and that it can occur subsequent to Zip1 dissociation from chromosome cores.

Entry into anaphase I is not required for Zip1 degradation and SC disassembly in the ipl1-mn mutant

In the pachytene arrest-and-release experiments, Zip1 eventually dissociated from the chromosomes in the majority of nuclei in the ipl1-mn mutant (Fig. 5D). Consistent with this, only $20 \%$ of anaphase I nuclei, compared with $80 \%$ of metaphase I nuclei, contained Zipl staining in the ipl1-mn mutant (Fig. 2E). Since metaphase I spindles can precociously elongate when homologous chromosomes fail to counteract the pulling forces (Visintin et al. 1998), we ascertained that Cdc14$13 \mathrm{Myc}$, which is released from the nucleolus at the onset of anaphase I, was not sequestered in the cells containing anaphase I spindles (Supplemental Fig. S9).

To examine whether the SC dissociation in anaphase I nuclei of ipl1-mn cells requires entry into anaphase I, we examined Zip1 dissociation in cells lacking Cdc20, an activator of the APC important for anaphase I entry (Schwab et al. 1997; Visintin et al. 1997; Shirayama et al. 1998). cdc20-mn NDT80-IN or cdc20-mn ipl1-mn NDT80-IN cells formed metaphase I spindles with a single nucleus, and no anaphase I spindles were observed; however, more "stretched" nuclei were observed for cdc20-mn ipl1-mn (data not shown). Zip1 removal from meiotic chromosomes and Zip1 degradation occurred with normal timing after pachytene release of $c d c 20-m n$ cells (Fig. 5I,J), and cdc20-mn ipl1-mn double mutants removed and degraded Zip1 with the same timing as ipl1-mn single mutants (Fig. $5 \mathrm{C}, \mathrm{K}, \mathrm{L})$. This indicates that the delayed dissociation of Zip1 in ipl1-mn mutants is independent of anaphase I entry, but may be correlated with the increased nuclear "stretching" observed.

\section{Behavior of axial element components and Zip1-interacting partners}

Smt3. Zip1 has been proposed to bind to sumoylated proteins associated with the lateral elements. In the wild type, Smt3 is observed along chromosome axes in pachytene nuclei (Fig. 6; Cheng et al. 2006; Hooker and Roeder 2006). By diplotene, Smt3 staining becomes less linear, consistent with chromosomes becoming more diffuse. This behavior is different from that of Zip1 (Fig. 2A). As chromosomes condense again before metaphase I, Smt 3 is found as thin, short stretches in $\sim 40 \%$ of the nuclei and as short stretches interspersed with foci in another $\sim 40 \%$ (Fig. 6A,B). By anaphase I, $>70 \%$ of nuclei contain short stretches, interspersed with foci (Fig. 6A). No substantial aggregation of Smt3 was observed at any of the stages in the wild type (Fig. 6C). Consistent with Zip1 dissociating from meiotic chromosomes prior to $\mathrm{Smt} 3$, costaining of both proteins demonstrated that by diplotene and metaphase I, most Zip1 had been removed from chromosome arms despite Smt3 still being present (Fig. 6G; Supplemental Fig. S10).

In the ipl1-mn mutant, a significant proportion of diplotene nuclei contained linear $\mathrm{Smt} 3$ staining that was different from the short stretches observed in the wild type. Not only were the stretches longer, they also appeared thicker, as in pachytene (Fig. 6D). Thirty percent of nuclei contained aggregates of Smt3 (Fig. 6F). At metaphase I as well as anaphase I, $\sim 80 \%$ of nuclei contained linear Smt3 staining, often with aggregates of Smt3 (Fig. 6E,F). Both the linear Smt3 as well as aggregates costained for Zip1 (Fig. 6H). These observations suggest altered association of Smt3 with chromosome cores at the G2/M transition in the ipl1-mn mutant.

Red1. Red1 is a component of axial elements and is found in discontinuous stretches, resembling "beads on a string" (Smith and Roeder 1997). We refer to these as short lines. Red1 dissociated from the meiotic chromosomes and was degraded with kinetics similar to Zip1 in the wild type (Fig. 7 ), although $\sim 50 \%$ of nuclei showed discrete foci at metaphase I. Furthermore, in a subset of nuclei that contained metaphase I spindles (one-third), Red1 was observed to be juxtaposed with the spindle poles (Supplemental Fig. S11). Redl protein levels were undetectable $\sim 90 \mathrm{~min}$ after pachytene release (Fig. 7G), similar to Zip1 (Fig. 5A).

In the ipl1-mn mutant, Red1 was retained as foci at diplotene, and a proportion ( $30 \%)$ of metaphase I nuclei contained short lines of Red1 (Fig. 7E). The majority of anaphase I nuclei did not contain any significant Red1 staining, and the one-third that did contained aggregates of Red1 (Fig. 7F). Instead of being associated with the spindle poles, these aggregates were found at the center of the anaphase I spindles (Supplemental Fig. S11). Overall, Red1 staining was significantly different from Smt3, although its behavior is also affected in the ipl1-mn mutant.

Red1 protein degradation occurred with only a slight delay in the ipl1-mn mutant (15-30 min) (Fig. 7H). Since Red1 is sumoylated (Cheng et al. 2006), Red1-Smt3 has been proposed to be a binding partner for Zip1. However, our cytological observations, supported by the Western blot analysis showing that Red1 protein levels are depleted more or less normally in ip11-mn (Fig. 7), suggest that it is unlikely that sumoylated Red1 is the main binding partner for Zip1, at least after SC assembly.

Hop1. Like Red1 and Smt3, Hop1 and Rec8 are important for Zip1 localization to meiotic chromosomes. The 
Jordan et al.
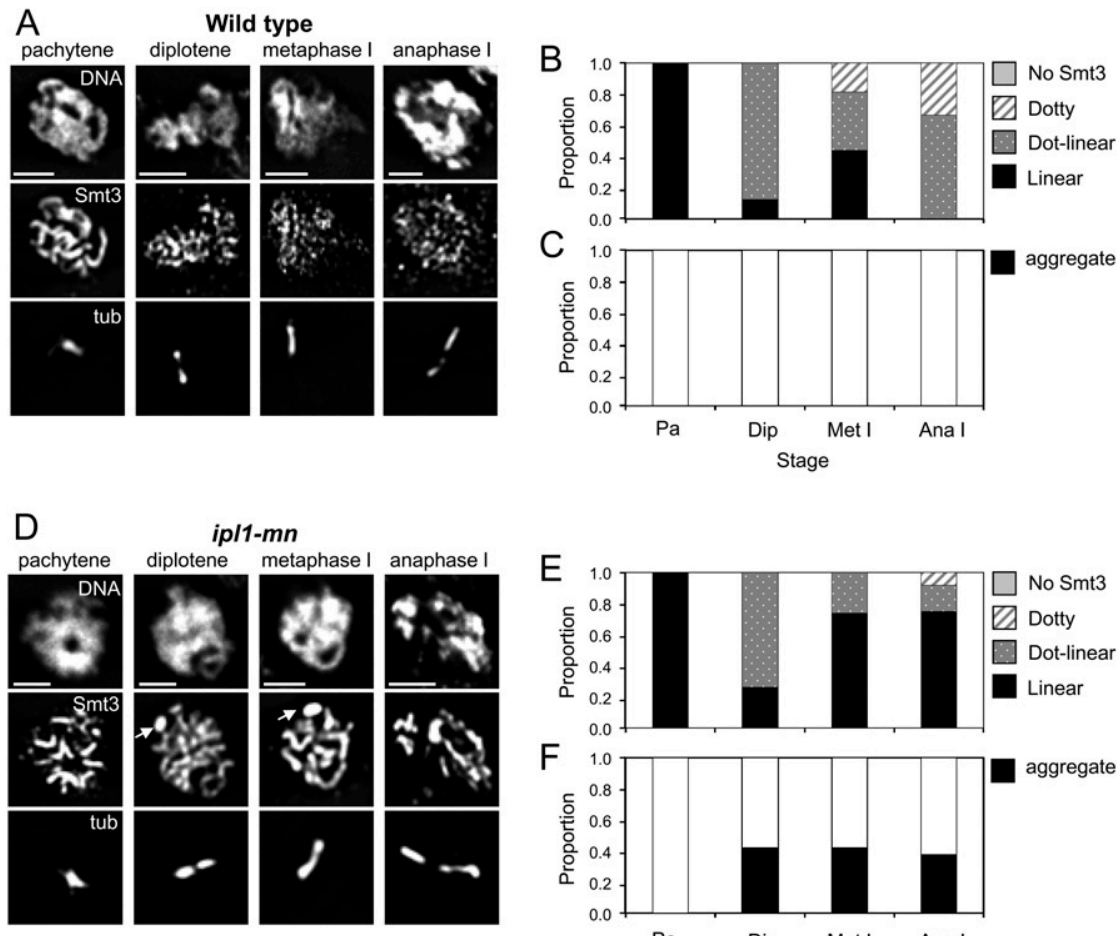

Figure 6. Aberrant association of $\mathrm{Smt} 3 /$ SUMO with meiotic chromosomes in the ipl1-mn mutant. $(A-C)$ Examples of Smt3 staining at different stages of meiosis I $(A)$ and quantification of Smt3 staining along chromosomes $(B)$ as well as accumulation of aggregates $(C)$ in the wild type. $(D)$ Aberrant behavior of Smt3 in ipl1-mn at diplotene-anaphase I. $(E, F)$ Quantification of nuclear staining $(E)$ and aggregate formation $(F)$. More than 50 nuclei were scored for each stage. $(G, H)$ Dual staining of Zip1 (green) and Smt3 (magenta) in wild-type (diplotene and metaphase I, $G$ ) and in the ipl1-mn mutant (metaphase I and anaphase I, H). Arrows indicate aggregates of Smt3 and Zip1. (tub) Tubulin. Bars, $2 \mu \mathrm{m}$. Singlechannel images for Zip1 and Smt3 are shown in Supplemental Figure S10. Strains: Y940 (wild type) and Y1206 (ipl1-mn).

G

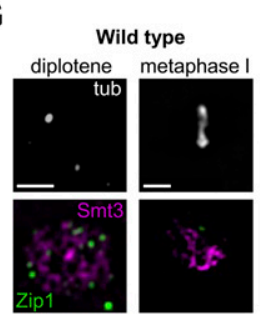

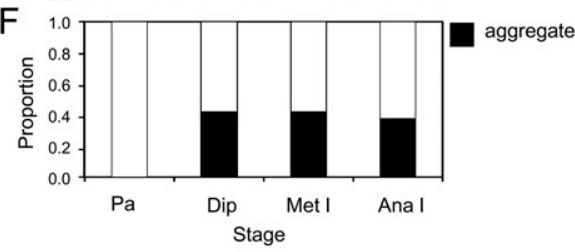

$\mathrm{H}$

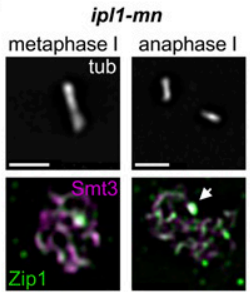

Hop1 staining pattern was similar in the wild type and the ipl1-mn mutant. Hop1 remained associated with the meiotic chromosomes after pachytene exit as multiple, distinct foci before appearing linear again in metaphase I and anaphase I (Supplemental Fig. S12). The persistence of Hopl on meiotic chromosomes is consistent with findings that protein levels remain high, even as cells undergo the meiotic nuclear divisions (Carballo et al. 2008).

Rec8. Rec8 was present as linear structures until the metaphase-anaphase I transition in both the wild type and ipl1-mn mutant (data not shown), as reported previously (Yu and Koshland 2007). When we assessed spreads with two clearly separated nuclei (late anaphase I/telophase I), the characteristic pattern of Rec8 staining at the poles (presumably at centromeres) of the spindle was observed in the wild type. We also observed some Zip1 staining (Supplemental Fig. S13A). In contrast, in anaphase I/telophase I spreads with retained Zip1 and two clearly separated nuclei in the ipl1-mn mutant, Rec8 was also present (30 of 30 nuclei) and, generally, colocalized with Zip1 (Supplemental Fig. S13B).
Regulation of chromosome dynamics by Ipl1 does not require histone $\mathrm{H} 3$ phosphoryation on Ser10

Ipl1 phosphorylates histone $\mathrm{H} 3$ on Ser10 throughout meiosis I prophase (Supplemental Fig. S1; Monje-Casas et al. 2007). However, this phosphorylation event is dispensable for SC disassembly (Supplemental Fig. S14), indicating that other targets of Ipl1's kinase are important for SC disassembly.

\section{Discussion}

SC disassembly in budding yeast: Smt3 and Hop1 remain associated with meiotic chromosomes, while Zip1 localizes to the centromeres and Red1 dissociates at pachytene exit

Here, we investigated SC disassembly in budding yeast. At pachytene exit, both the central element component Zip1 and the lateral element component Red1 dissociate from the meiotic chromosomes and are degraded (Figs. 1, 2, 4, 6). Some Zip1 protein remains associated with centromeres and has been shown to be important for 

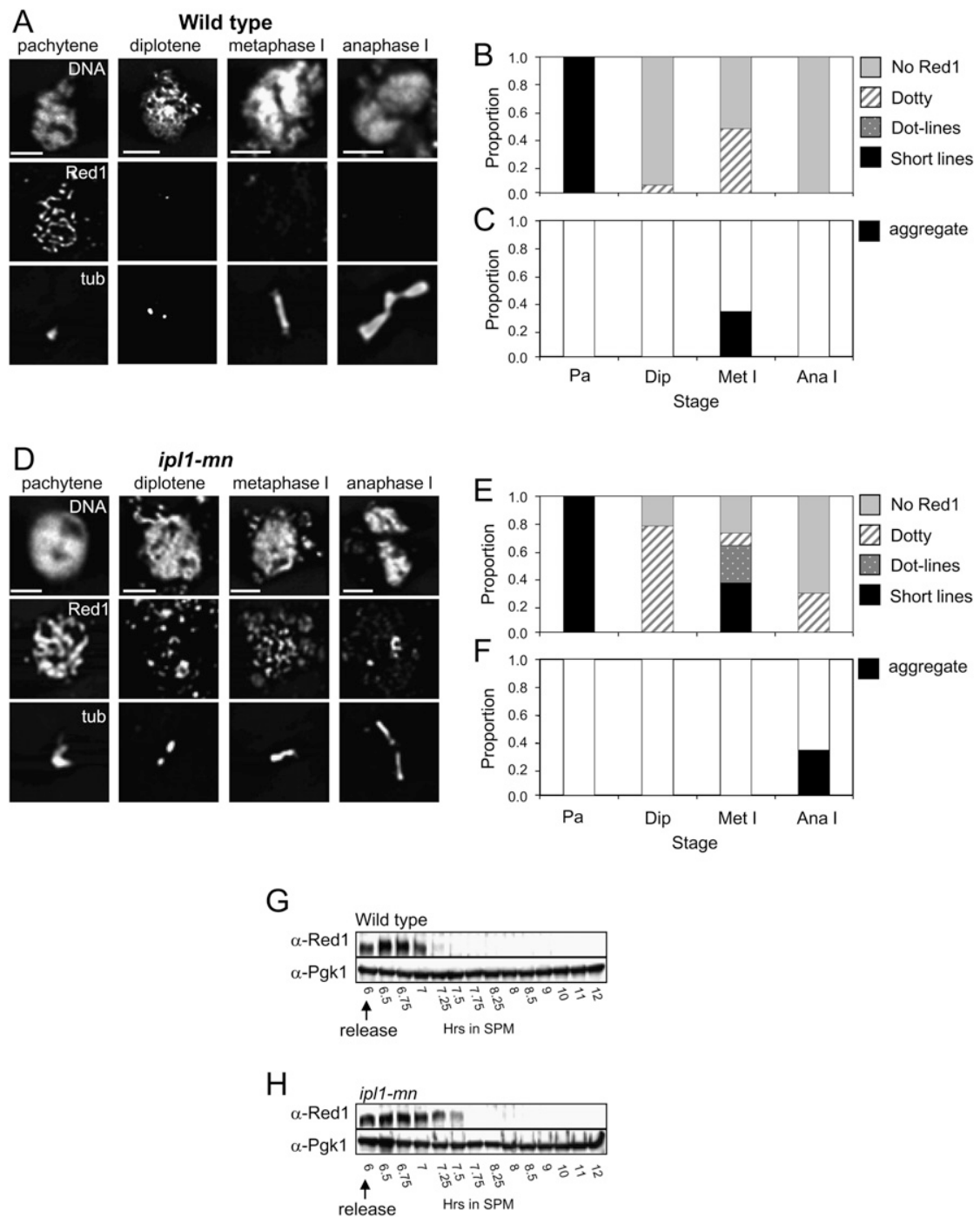

Figure 7. Redl dissociation from meiotic chromosomes and its degradation are slightly delayed in the ipl1-mn mutant. Examples of Red1 staining in nuclei at various stages of meiosis I in wild type $(A)$ and ipl1-mn $(D)$. (tub) Tubulin. Bars, $2 \mu \mathrm{m}$. Note that Redl staining is not continuous along chromosomes, while Zip1 and Smt3 are. Quantification of Redl staining of meiotic chromosomes and aggregate formation in wild type $(B, C)$ and ipl1-mn $(E, F)$. More than 50 nuclei were scored for each stage. Y940 (wild type) and Y1206 (ipl1-mn). $(G, H)$ Western blot analysis of Redl protein levels after pachytene release. Strains: Y1602 (wild type) and Y1538 (ip11-mn).

nonexchange chromosome segregation (L Newnham, P Jordan, B Rockmill, S Roeder, and E Hoffmann, in prep.).

In contrast, Smt3/SUMO and Hop1 remain associated with the meiotic chromosomes as foci and short stretches even after the first meiotic division (Fig. 5; Supplemental Fig. S12). Thus, one protein known to interact with Zip1 (Smt3/SUMO) during SC assembly (Cheng et al. 2006) is retained after the dissociation of Zip1. This is different from the pattern observed during SC assembly, when Smt3 and Zip1 staining occur concomitantly (Hooker and Roeder 2006).

\section{A role for Ipl1 in SC disassembly prior to its functions in $M$ phase}

Ipl1 has been found previously to have important roles in chromosome segregation during both meiosis I and meiosis II (Monje-Casas et al. 2007; Yu and Koshland 2007). Here, we identified a new function for Ipl1 in promoting SC disassembly (Figs. 1, 2), which precedes Ipl1's role in ensuring accurate chromosome segregation during $M$ phase. Both components of the axial elements (Red1 and Smt3) as well as the central element (Zip1) display delayed dissociation from the meiotic chromosome cores in the ipl1-mn mutant. The delayed disassembly of the SC raises the possibility that the chromosome missegregation frequency of ipl1-mn mutants could be caused by its retention. This does not appear to be the case, since a homolog pair missegregates with similar frequencies in ipl1-mn and ipl1-mn zip1 mutants ( 80\%) (Monje-Casas et al. 2007; data not shown).

\section{Dissociation of central and lateral element components are delayed in ipl1-mn}

Both disassembly of lateral element components as well as the central element component were affected in the ipl1-mn mutant. In the ipl1-mn mutant, Smt3 was observed as linear stretches after pachytene exit (Fig. 5). These, as well as Smt3 aggregates, colocalized with Zip1 in the ipl1-mn mutant (Fig. 5H), consistent with the model that Smt3 is a substrate of Zip1 (Cheng et al. 2007).

Red1, a lateral element component, showed slightly delayed dissociation from the chromosomes. However, 
Red1 protein was eventually degraded ( 30-min delay) (Fig. 6). Thus, the behavior of the central element component (Zip1) and its interacting partner (Smt3), as well as a lateral element component (Red1), are affected in the absence of Ipl1.

Two other lateral element components, Hop1 and Rec8, remain associated with the chromosome cores in wild type until the metaphase I-anaphase I transition and could be candidates for linking Zip1 to chromatin. For instance, the correlation and colocalization between Rec8 and Zip1 in anaphase I nuclei of ipl1-mn makes Rec8 a potential candidate (Supplemental Fig. S13B).

Given that Red1, Rec8, and Smt3 dissociation with the meiotic chromosomes is affected in the ipl1-mn mutant, we considered the possibility that Ipll might induce several changes to chromosome structure. One of the best-characterized targets of Ipl1 kinase is histone H3. Phosphorylation of histone $\mathrm{H} 3$ at Ser10 correlates with chromosome condensation in a variety of organisms, including budding yeast. During meiosis, Ser10 phosphorylation occurs at its highest levels between 3 and $6 \mathrm{~h}$ in meiosis (Supplemental Fig. S1), and chromosome spreads show costaining of phosphorylated histone H3 with Zip1 from zygotene to pachytene (Hsu et al. 2000). The role of this phosphorylation event, however, is unclear. Although Tetrahymena micronuclei mutated in Ser10 H3 show defects in chromosome condensation and meiotic transmission (Wei et al. 1998, 1999), budding yeast mutants lacking the target serine do not show any defects in mitotic or meiotic chromosome transmission /data not shown), consistent with previous observations (Hsu et al. 2000). We failed to observe any changes to SC disassembly in a strain expressing a histone H3 Ser10-Ala mutant protein (Supplemental Fig. S14). Thus, phosphorylation of histone H3 Ser10 is dispensable for SC disassembly in budding yeast. This conclusion is consistent with those made in studies of mouse spermatogenesis, where Aurora B kinase phosphorylation of histone H3 Ser10 occurs after the dissociation of the central element component SYCP1 (Sun and Handel 2008).

Our observations have some parallels with those made in Drosophila oocytes, where NHK-1 promotes SC disassembly of both central and lateral elements (Ivanovska et al. 2005). However, our observations differ from those made during mouse spermatogenesis. While inhibition of the Aurora kinases causes the retention of the lateral element component SYCP3, it does not prevent the dissociation of the central element SYCP1. Interestingly, in wild-type spermatogenesis, SYCP3 dissociates with similar characteristics to Zip1 in budding yeast, disassembling during late prophase I and remaining associated with the centromeric regions (Sun and Handel 2008).

\section{Ipl1 ties SC disassembly to cell cycle progression and joint molecule resolution}

Several cytological and biochemical observations support the conclusion that Ipl1/Aurora B kinase links SC disassembly to the G2/M transition in budding yeast. First, linear SCs are present in $\sim 80 \%$ of metaphase I nuclei from an ipl1-mn mutant (Fig. 2E), whereas no such nuclei were observed in wild type (Fig. 2B). Second, although S-phase completion is delayed $\sim 30-60 \mathrm{~min}$ in the ipl1-mn mutant (Fig. 3A), subsequent cell cycle events, including SPB separation (Fig. 3B), are also similarly delayed in the ipl1-mn mutant (Fig. 3). This suggests that cell cycle progression occurs with similar kinetics as in wild type. Thus, the simplest explanation to account for the apparent decoupling of cell cycle progression (i.e., SPB separation and metaphase I spindle formation) and SC disassembly is that Ipl1 promotes the timely dissociation of Zip1 from the meiotic chromosomes at the end of pachytene.

One of the key events occurring at the end of pachytene is the resolution of joint molecules to crossovers. The ipl1-mn mutant showed normal levels of DSB formation and repair to both crossovers and noncrossovers (Fig. 3; Supplemental Fig. S8). Therefore, the retention of Zip1 on the chromosome does not appear to affect crossover formation. We conclude that Ipll ensures that full SC disassembly occurs at the same time as joint molecule resolution.

Four kinases regulate major events at the end of meiotic prophase

Our data show that Ipl1 links SC disassembly to both cell cycle progression and joint molecule resolution. In budding yeast, CDK1-M promotes SPB separation and the formation of metaphase I spindles. Neither is observed when the Cdc28 kinase subunit is inhibited, although SC disassembly and Zip1 degradation do occur (Sourirajan and Lichten 2008). Furthermore, joint molecule resolution is also not inhibited when Cdc28 is inactivated (Sourirajan and Lichten 2008). Rather, joint molecule resolution is promoted by Cdc5/Polo-like kinase. cdc5mn mutants show high levels of joint molecule accumulation and low levels of crossovers, suggesting that Cdc5 activity is important for joint molecule resolution /Clyne et al. 2003). While Cdc5 expression is sufficient to pro-

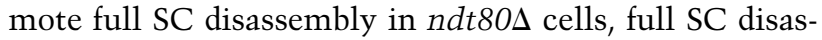
sembly can occur in the absence of Cdc5 (Fig. 4). This is promoted by Ipl1, since the ipl1-mn cdc5-mn double mutant showed delayed SC disassembly, similar to ipl1$m n$. Furthermore, ipl1-mn causes retention of SCs when CDC5 expression is induced (Fig. 4). Our observations suggest that there are multiple mechanisms for SC disassembly. On the other hand, neither cell cycle progression nor joint molecule resolution is affected in the ipl1-mn mutant.

Collectively, these observations lead us to propose that four different kinases promote distinct events upon pachytene exit. CDK1-M ensures proper spindle behavior, Cdc5 promotes joint molecule resolution, Ipl1 stimulates SC disassembly, and Cdc7 as well as Cdc5 enforces mono-orientation of sister kinetochores /Clyne et al. 2003; Lee and Amon 2003; Lo et al. 2008; Matos et al. 2008).

It has been suggested previously that joint molecule resolution could promote timely SC disassembly (Sourirajan 
and Lichten 2008). Although the $c d c 5-m n$ mutant displayed a relatively minor SC disassembly phenotype compared with ipl1-mn (Supplemental Table S1), the majority of nuclei at diplotene or beyond contained many foci of Zip1. These were more numerous than in wild type (data not shown), where Zip1 is retained at centromeres after pachytene exit (L Newnham, P Jordan, B Rockmill, S Roeder, and E Hoffmann, in prep.). It is possible that these Zip1 foci mark the sites of unresolved joint molecules. If so, then SC disassembly at sites of crossovers may be distinct from general removal of Zip1 from the arm regions. Currently, we do not have any cytological markers that allow us to identify (and therefore distinguish between) crossover products or joint molecules and correlate this transition with removal of Zip1 from the meiotic chromosomes on a per nucleus basis.

\section{Materials and methods}

\section{Sporulation conditions}

The strains used are listed in the Supplemental Material (Supplemental Table S2). Diploid cells were grown to saturation in YEPD $(1 \%$ yeast extract, $2 \%$ bactopeptone, $2 \%$ dextrose at $\mathrm{pH} 6.5)$, then inoculated at $5 \times 10^{6}$ cells per milliliter in SPS $(0.05 \%$ yeast extract, $1 \%$ peptone, $0.17 \% \mathrm{YNB}, 1 \%$ potassium acetate [KAc], $0.5 \%$ ammonium sulfate, $0.05 \mathrm{M}$ potassium hydrogen pthalate at $\mathrm{pH}$ $5.5)$ and grown until a cell density of $5 \times 10^{7}$ cells per milliliter had been reached, before being resuspended in $1 \%$ potassium acetate, supplemented with $0.02 \%$ raffinose and $0.02 \%$ antifoam (pH 7.0) (Sigma, A8311). For S288c, cells were resuspended in YPA ( $1 \%$ yeast extract, $2 \%$ bacto-peptone, $2 \% \mathrm{KAc}$ ) and grown to $5 \times 10^{7}$ to $5.5 \times 10^{7}$ cells per milliliter before being induced to undergo meiosis in $2 \% \mathrm{KAc}$ and $0.02 \%$ raffinose $(\mathrm{pH} 7.0)$.

\section{Indirect immunofluorescence on whole cells}

Indirect immunofluorescence was carried out by fixing 4-8 mL of cells $\left(\sim 2 \times 10^{7}\right.$ cells per milliliter $)$ in $4 \%$ formaldehyde for 15-60 min at room temperature. Cells were harvested and resuspended in $1 \mathrm{~mL}$ of IS buffer $(1.2$ M sorbitol, $100 \mathrm{mM} \mathrm{K} \mathrm{HPO}_{4}, 100 \mathrm{mM} \mathrm{KH} \mathrm{PO}_{4}, 100 \mathrm{mM}$ $\mathrm{MgCl}_{2}$ ). Cells were transferred to an Eppendorf tube and washed three times in IS buffer before being resuspended in $100 \mu \mathrm{L}$ of IS buffer containing $2 \mu \mathrm{L}$ of DTT $(1 \mathrm{M})$ and $10 \mu \mathrm{L}$ of zymolyase $100 \mathrm{~T}(10 \mathrm{mg} / \mathrm{mL}$; Seiguku Corporation). The cells were incubated at $37^{\circ} \mathrm{C}$ until they had lost most of the cell wall and contained "rough" edges. Cells were washed with $100 \mu \mathrm{L}$ of PBS-N-N containing PBS $+150 \mathrm{mM} \mathrm{NaCl}$ and $50 \mathrm{mM} \mathrm{Na} \mathrm{PO}_{4}$ (pH 7.4), gently pelleted, and resuspended in $67 \mu \mathrm{L}$ of PBS$\mathrm{N}-\mathrm{N}+0.1 \% \mathrm{NP} 40$. After $30 \mathrm{~min}$ incubation at room temperature, $33 \mu \mathrm{L}$ of fetal bovine serum containing the appropriate primary antibodies were added, and cells were incubated for $4 \mathrm{~h}$ at room temperature and then overnight at $4{ }^{\circ} \mathrm{C}$. Cells were washed three times in $1 \mathrm{~mL}$ of PBS-N-N and resuspended in 50-100 $\mu \mathrm{L}$ of mounting solution containing DAPI.

\section{Meiotic surface spread nuclei}

Surface spread nuclei were prepared as described (Chua and Roeder 1998). Primary antibodies were used in the following concentrations: rat $\alpha$-tubulin (1:1000; Novus Biologicals), rabbit $\alpha$-Zip1 (1:200; Eurogentec), goat $\alpha$-Zip1 (1:100; Santa Cruz Biotechnologies, sc-15632), and rabbit$\alpha$-Smt3 (1:100; Santa Cruz Biotechnologies, sc-28649). Secondary antibodies were obtained for Stratech and used 1:200. To identify meiotic nuclei at pachytene, we used highly condensed "worm-like" DNA and/or linear Zip1 staining. For subsequent stages, we used tubulin to assess SPB behavior. The diffuse stage was characterized by juxtaposed SPBs and diffuse DNA, whereas the diplotene stage was identified by a single body of DNA containing separated SPBs. For metaphase I, we used "club-like" spindles $\sim 2 \mu \mathrm{m}$, and for anaphase I we used spindles $>4 \mu \mathrm{m}$.

\section{Western blot analysis}

Proteins samples were prepared by post-alkaline extraction (Kushnirov 2000) or TCA extraction (Clyne et al. 2009). Cells from $2.5 \mathrm{~mL}$ of liquid culture were pelleted and resuspended in $100 \mu \mathrm{L}$ of $0.1 \mathrm{M} \mathrm{NaOH}$, incubated for $5 \mathrm{~min}$ at room temperature, and resuspended in modified Lamaelli sample buffer $(0.06 \mathrm{M}$ Tris-Hcl at $\mathrm{pH} 6.8$, $5 \%$ glycerol, $2 \%$ SDS, $4 \% \beta$-mercaptoethanol, $0.0025 \%$ bromophenol blue). Protein samples were separated on SDS-PAGE gels, transferred to a nitrocellulose membrane, and probed with the appropriate primary antibodies, which included a goat $\alpha$-Zip1 (1:2000; Santa Cruz Biotechnologies, sc-15632), rabbit $\alpha$-Histone H3 (Millipore, 05-928, 1:2000), rabbit p-Histone H3 (Ser10)R (1:2000; Santa Cruz Biotechnologies, sc-8656-R), rabbit $\alpha$-Red1 (1:2000; Roeder Laboratories) (Smith and Roeder 1997), mouse 12CA5 $\alpha$-HA (1:1000; CRUK), and $\alpha$-3-PGK as a loading control (Molecular Probes, A-6457) at $1: 10,000$. The goat $\alpha$-Zip1 IgG was detected with HRPconjugated rabbit $\alpha$-goat IgG (DakoCytomation, P0449), mouse $\alpha$-3-PGK was detected with HRP-conjugated rabbit $\alpha$-mouse IgG (DakoCytomation, P0260), and the antibodies raised in rabbit were detected with HRP-conjugated swine $\alpha$-rabbit IgG (DakoCytomation, P0399). All secondary antibodies were diluted 1:2000. PerkinElmer Western Lightning Chemiluminescence Reagent and Fuji Super RX X-ray film were used for development. Protein levels were determined using ImageJ.

\section{Purification of antibodies from serum}

To purify IgGs from serum raised against Hop 1 and Red1 proteins (gifts from Dr. Franz Klein and Professor G. Shirleen Roeder, respectively), Protein A beads/resin (Sigma) were washed once in PBS and resuspended in twice the volume of PBS (50\% resin). One-hundred microliters of the $50 \%$ resin were incubated with 200 $\mu \mathrm{L}$ of serum for $1 \mathrm{~h}$ at $4^{\circ} \mathrm{C}$ while rotating. This was followed by four washes in $2 \mathrm{~mL}$ of $0.1 \times \mathrm{PBS}$ (diluted with water). IgGs were eluted in $0.2 \mathrm{M}$ glycine (pH 2.5) (Fisher). All steps were carried out at $4^{\circ} \mathrm{C}$ or with samples on ice. 
Jordan et al.

\section{Southern blot analysis}

Southern blotting was carried out as described (Allers and Lichten 2001). ImageJ (NIH) was used to quantify DNA levels.

\section{Acknowledgments}

We thank Angelika Amon, Min-Hao Kuo, Franz Klein, and G. Shirleen Roeder for antibodies and strains, and members of the Hoffmann and Lichten laboratories for discussion and comments on this work. The Hoffmann laboratory was funded by a grant from the Medical Research Council and the Lichten laboratory was funded by an Intramural Research Program of the National Cancer Institute, NIH. E.H. was funded by a Royal Society Dorothy Hodgkin Fellowship.

\section{References}

Allers T, Lichten M. 2001. Differential timing and control of noncrossover and crossover recombination during meiosis. Cell 106: 47-57.

Benjamin KR, Zhang C, Shokat KM, Herskowitz I. 2003. Control of landmark events in meiosis by the CDK Cdc28 and the meiosis-specific kinase Ime2. Genes \& Dev 17: 1524-1539.

Bhalla N, Dernburg AF. 2008. Prelude to a division. Annu Rev Cell Dev Biol 24: 397-424.

Bishop AC, Ubersax JA, Petsch DT, Matheos DP, Gray NS, Blethrow J, Shimizu E, Tsien JZ, Schultz PG, Rose MD, et al. 2000. A chemical switch for inhibitor-sensitive alleles of any protein kinase. Nature 407: 395-401.

Borde V, Goldman AS, Lichten M. 2000. Direct coupling between meiotic DNA replication and recombination initiation. Science 290: 806-809.

Borner GV, Kleckner N, Hunter N. 2004. Crossover/noncrossover differentiation, synaptonemal complex formation, and regulatory surveillance at the leptotene/zygotene transition of meiosis. Cell 117: 29-45.

Brar GA, Hochwagen A, Ee LS, Amon A. 2009. The multiple roles of cohesin in meiotic chromosome morphogenesis and pairing. Mol Biol Cell 20: 1030-1047.

Carballo JA, Johnson AL, Sedgwick SG, Cha RS. 2008. Phosphorylation of the axial element protein Hop1 by Mec1/Tel1 ensures meiotic interhomolog recombination. Cell 132: 758770.

Carlile TM, Amon A. 2008. Meiosis I is established through division-specific translational control of a cyclin. Cell 133: 280-291.

Chen SY, Tsubouchi T, Rockmill B, Sandler JS, Richards DR, Vader G, Hochwagen A, Roeder GS, Fung JC. 2008. Global analysis of the meiotic crossover landscape. Dev Cell 15: 401-415.

Cheng CH, Lo YH, Liang SS, Ti SC, Lin FM, Yeh CH, Huang HY, Wang TF. 2006. SUMO modifications control assembly of synaptonemal complex and polycomplex in meiosis of Saccharomyces cerevisiae. Genes \& Dev 20: 2067-2081.

Cheng $\mathrm{CH}$, Lin FM, Lo YH, Wang TF. 2007. Tying SUMO modifications to dynamic behaviors of chromosomes during meiotic prophase of Saccharomyces cerevisiae. J Biomed Sci 14: 481-490.

Cheslock PS, Kemp BJ, Boumil RM, Dawson DS. 2005. The roles of MAD1, MAD2 and MAD3 in meiotic progression and the segregation of nonexchange chromosomes. Nat Genet 37: $756-760$.

Chu S. 1998. The transcriptional program of sporulation in budding yeast. Science 282: 1421 .
Chu S, Herskowitz I. 1998. Gametogenesis in yeast is regulated by a transcriptional cascade dependent on Ndt80. Mol Cell 1: 685-696.

Chu S, DeRisi J, Eisen M, Mulholland J, Botstein D, Brown PO, Herskowitz I. 1998. The transcriptional program of sporulation in budding yeast. Science 282: 699-705.

Chua PR, Roeder GS. 1998. Zip2, a meiosis-specific protein required for the initiation of chromosome synapsis. Cell 93: 349-359.

Clyne RK, Katis VL, Jessop L, Benjamin KR, Herskowitz I, Lichten M, Nasmyth K. 2003. Polo-like kinase Cdc5 promotes chiasmata formation and cosegregation of sister centromeres at meiosis I. Nat Cell Biol 5: 480-485.

Clyne M, Offman J, Shanley S, Virgo JD, Radulovic M, Wang Y, Ardern-Jones A, Eeles R, Hoffmann E, Yu VP. 2009. The G67E mutation in hMLH1 is associated with an unusual presentation of Lynch syndrome. Br J Cancer 100: 376380.

Colaiacovo MP. 2006. The many facets of SC function during $C$. elegans meiosis. Chromosoma 115: 195-211.

Henderson KA, Keeney S. 2005. Synaptonemal complex formation: Where does it start? Bioessays 27: 995-998.

Hooker GW, Roeder GS. 2006. A Role for SUMO in meiotic chromosome synapsis. Curr Biol 16: 1238-1243.

Hsu JY, Sun ZW, Li X, Reuben M, Tatchell K, Bishop DK, Grushcow JM, Brame CJ, Caldwell JA, Hunt DF, et al. 2000. Mitotic phosphorylation of histone H3 is governed by Ipl1/ aurora kinase and Glc7/PP1 phosphatase in budding yeast and nematodes. Cell 102: 279-291.

Hunter N, Kleckner N. 2001. The single-end invasion: An asymmetric intermediate at the double-strand break to double-holliday junction transition of meiotic recombination. Cell 106: 59-70.

Ivanovska I, Khandan T, Ito T, Orr-Weaver TL. 2005. A histone code in meiosis: The histone kinase, NHK-1, is required for proper chromosomal architecture in Drosophila oocytes. Genes \& Dev 19: 2571-2582.

Kelly AE, Funabiki H. 2009. Correcting aberrant kinetochore microtubule attachments: An Aurora B-centric view. Curr Opin Cell Biol 21: 51-58.

Kushnirov VV. 2000. Rapid and reliable protein extraction from yeast. Yeast 16: 857-860.

Lee BH, Amon A. 2003. Role of Polo-like kinase CDC5 in programming meiosis I chromosome segregation. Science 300: 482-486.

Lo HC, Wan L, Rosebrock A, Futcher B, Hollingsworth NM. 2008. Cdc7-Dbf4 regulates NDT80 transcription as well as reductional segregation during budding yeast meiosis. Mol Biol Cell 19: 4956-4967.

Matos J, Lipp JJ, Bogdanova A, Guillot S, Okaz E, Junqueira M, Shevchenko A, Zachariae W. 2008. Dbf4-dependent CDC7 kinase links DNA replication to the segregation of homologous chromosomes in meiosis I. Cell 135: 662-678.

Monje-Casas F, Prabhu VR, Lee BH, Boselli M, Amon A. 2007. Kinetochore orientation during meiosis is controlled by Aurora B and the Monopolin Complex. Cell 128: 477490.

Parra MT, Viera A, Gomez R, Page J, Carmena M, Earnshaw WC, Rufas JS, Suja JA. 2003. Dynamic relocalization of the chromosomal passenger complex proteins inner centromere protein (INCENP) and aurora-B kinase during male mouse meiosis. J Cell Sci 116: 961-974.

Parra MT, Gomez R, Viera A, Llano E, Pendas AM, Rufas JS, Suja JA. 2009. Sequential assembly of centromeric proteins in male mouse meiosis. PLoS Genet 5: e1000417. doi: 10.1371/ journal.pgen.1000417. 
Petronczki M, Siomos MF, Nasmyth K. 2003. Un menage a quatre: The molecular biology of chromosome segregation in meiosis. Cell 112: 423-440.

Pinsky BA, Kung C, Shokat KM, Biggins S. 2006. The Ipl1Aurora protein kinase activates the spindle checkpoint by creating unattached kinetochores. Nat Cell Biol 8: 78-83.

Riedel CG, Katis VL, Katou Y, Mori S, Itoh T, Helmhart W, Galova M, Petronczki M, Gregan J, Cetin B, et al. 2006. Protein phosphatase $2 \mathrm{~A}$ protects centromeric sister chromatid cohesion during meiosis I. Nature 441: 53-61.

Rockmill B, Voelkel-Meiman K, Roeder GS. 2006. Centromereproximal crossovers are associated with precocious separation of sister chromatids during meiosis in Saccharomyces cerevisiae. Genetics 174: 1745-1754.

Roeder GS. 1997. Meiotic chromosomes: It takes two to tango. Genes \& Dev 11: 2600-2621.

Roeder GS, Bailis JM. 2000. The pachytene checkpoint. Trends Genet 16: 395-403.

Ruchaud S, Carmena M, Earnshaw WC. 2007. Chromosomal passengers: Conducting cell division. Nat Rev Mol Cell Biol 8: 798-812.

Schwab M, Lutum AS, Seufert W. 1997. Yeast Hct1 is a regulator of Clb2 cyclin proteolysis. Cell 90: 683-693.

Schwacha A, Kleckner N. 1995. Identification of double Holliday junctions as intermediates in meiotic recombination. Cell 83: 738-791.

Shirayama M, Zachariae W, Ciosk R, Nasmyth K. 1998. The Polo-like kinase Cdc5p and the WD-repeat protein Cdc20p/ fizzy are regulators and substrates of the anaphase promoting complex in Saccharomyces cerevisiae. EMBO I 17: 13361349 .

Smith AV, Roeder GS. 1997. The yeast Red1 protein localizes to the cores of meiotic chromosomes. J Cell Biol 136: 957967.

Sourirajan A, Lichten M. 2008. Polo-like kinase Cdc5 drives exit from pachytene during budding yeast meiosis. Genes \& Dev 22: $2627-2632$.

Storlazzi A, Xu L, Schwacha A, Kleckner N. 1996. Synaptonemal complex component Zip1 plays a role in meiotic recombination independent of SC polymerisation along the chromosomes. Proc Natl Acad Sci 93: 9043-9048.

Sun F, Handel MA. 2008. Regulation of the meiotic prophase I to metaphase I transition in mouse spermatocytes. Chromosoma 117: 471-485.

Sym M, Roeder GS. 1995. Zip1-induced changes in synaptonemal complex structure and polycomplex assembly. I Cell Biol 128: 455-466.

Sym M, Engebrecht JA, Roeder GS. 1993. ZIP1 is a synaptonemal complex protein required for meiotic chromosome synapsis. Cell 72: 365-378.

Tang Z, Shu H, Qi W, Mahmood NA, Mumby MC, Yu H. 2006. PP2A is required for centromeric localization of Sgol and proper chromosome segregation. Dev Cell 10: 575-585.

Toth A, Rabitsch KP, Galova M, Schleiffer A, Buonomo SB, Nasmyth K. 2000. Functional genomics identifies monopolin: A kinetochore protein required for segregation of homologs during meiosis I. Cell 103: 1155-1168.

Tsubouchi T, Roeder GS. 2005. A synaptonemal complex protein promotes homology-independent centromere coupling. Science 308: 870-873.

Tsubouchi T, Macqueen AJ, Roeder GS. 2008. Initiation of meiotic chromosome synapsis at centromeres in budding yeast. Genes \& Dev 22: 3217-3226.

Visintin R, Prinz S, Amon A. 1997. CDC20 and CDH1: A family of substrate-specific activators of APC-dependent proteolysis. Science 278: 460-463.
Visintin R, Craig K, Hwang ES, Prinz S, Tyers M, Amon A. 1998. The phosphatase Cdc14 triggers mitotic exit by reversal of Cdk-dependent phosphorylation. Mol Cell 2: 709-718.

Wei Y, Mizzen CA, Cook RG, Gorovsky MA, Allis CD. 1998. Phosphorylation of histone $\mathrm{H} 3$ at serine 10 is correlated with chromosome condensation during mitosis and meiosis in Tetrahymena. Proc Natl Acad Sci 95: 7480-7484.

Wei Y, Yu L, Bowen J, Gorovsky MA, Allis CD. 1999. Phosphorylation of histone $\mathrm{H} 3$ is required for proper chromosome condensation and segregation. Cell 97: 99-109.

Wu HY, Burgess SM. 2006. Two distinct surveillance mechanisms monitor meiotic chromosome metabolism in budding yeast. Curr Biol 16: 2473-2479.

Xu L, Ajimura M, Padmore R, Klein C, Kleckner N. 1995. NDT80, a meiosis-specific gene required for exit from pachytene in Saccharomyces cerevisiae. Mol Cell Biol 15: 6572-6581.

Yu HG, Koshland D. 2007. The Aurora kinase Ipll maintains the centromeric localization of PP2A to protect cohesin during meiosis. J Cell Biol 176: 911-918.

Zickler D, Kleckner N. 1999. Meiotic chromosomes: Integrating structure and function. Annu Rev Genet 33: 603-754.

Zickler D, Olson LW. 1975. The synaptonemal complex and the spindle plaque during meiosis in yeast. Chromosoma 50: 1-23. 


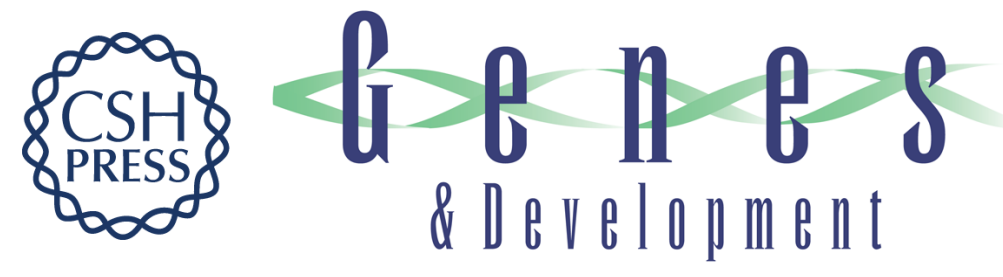

\section{Ipl1/Aurora B kinase coordinates synaptonemal complex disassembly with cell cycle progression and crossover formation in budding yeast meiosis}

Philip Jordan, Alice Copsey, Louise Newnham, et al.

Genes Dev. 2009, 23:

Access the most recent version at doi:10.1101/gad.536109

Supplemental http://genesdev.cshlp.org/content/suppl/2009/09/16/23.18.2237.DC1

Material

References This article cites 62 articles, 22 of which can be accessed free at:

http://genesdev.cshlp.org/content/23/18/2237.full.html\#ref-list-1

License

Email Alerting Receive free email alerts when new articles cite this article - sign up in the box at the top

Service right corner of the article or click here.

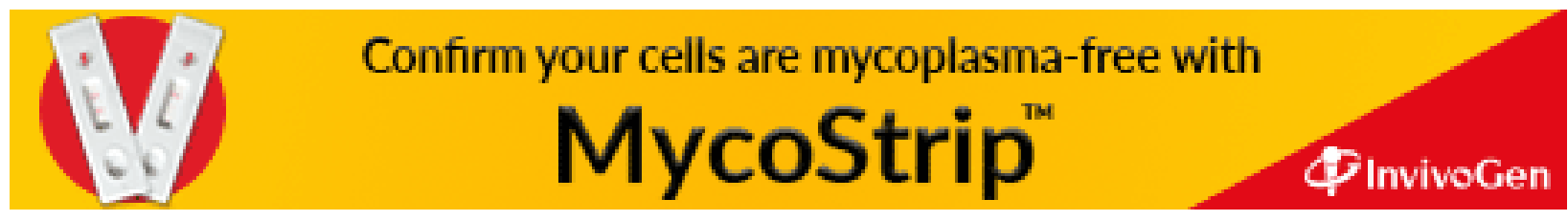

